\title{
Inattentional blindness in older adults: Effects of attentional set and to-be-ignored distractors
}

\author{
Sally Horwood ${ }^{1}$ - Vanessa Beanland ${ }^{1}$
}

Published online: 12 January 2016

(C) The Psychonomic Society, Inc. 2016

\begin{abstract}
Inattentional blindness (IB) involves failing to detect an unexpected visual stimulus while undertaking another task. Previous research has predominantly investigated IB using young adult samples, with few studies exploring whether or how an observer's age affects their detection of unexpected events. To help address this gap, we compared younger adults (18-25 years of age) and older adults (60-80 years of age) on two IB tasks: one dynamic, one static. In the static task, older age was associated with substantially increased IB rates: $89 \%$ for older adults versus $5 \%$ for younger adults. In the dynamic task, we systematically manipulated the presence of to-be-ignored distractors and whether the unexpected stimulus color matched the observers' attentional set. We found a main effect of age on IB: As in the static task, older age was associated with increased IB rates (38\% for older adults vs. $8 \%$ for younger adults). The presence of to-be-ignored distractors and attentional set mismatch interacted to substantially increase IB rates, but age did not interact with either factor. Overall, the results indicate that older age is associated with large increases in IB rates across a range of tasks. The pattern of results is consistent with attentional capacity models of cognitive aging, suggesting that older adults' reduced cognitive resources result in failure to consciously process stimuli that are inconsistent with their attentional set.
\end{abstract}

Keywords Aging $\cdot$ Visual awareness $\cdot$ Divided attention $\cdot$ Inattentional blindness

Vanessa Beanland

vanessa.beanland@anu.edu.au

1 Research School of Psychology, Australian National University, CanberraACT, 2601, Australia
Inattentional blindness (IB; Mack \& Rock, 1998) occurs when unexpected objects or events fail to reach conscious awareness because the observer is engaged in a cognitively or perceptually demanding task. Existing IB research and theories have largely focused on the conditions that produce IB, such as task demands and stimulus characteristics (e.g., Most, Scholl, Clifford, \& Simons, 2005; Most et al., 2001; Simons \& Jensen, 2009). This approach neglects differences between individuals and groups that may influence noticing rates. Recent IB research has increasingly examined characteristics that may influence an individual's susceptibility to IB, including domain expertise (Memmert, 2006), personality (Kreitz, Schnuerch, Gibbons, \& Memmert, 2015), autism (Swettenham et al., 2014), attention deficit hyperactivity disorder (Grossman, Hoffman, Berger, \& Zivotofsky, 2015), and cognitive abilities including working memory capacity (e.g., Beanland \& Chan, in press; Bredemeier \& Simons, 2012; Hannon \& Richards, 2010; Kreitz, Furley, Memmert, \& Simons, 2015; Richards, Hannon, \& Derakshan, 2010; Seegmiller, Watson, \& Strayer, 2011).

Relatively few studies have explicitly examined agerelated differences in IB, and most of these have focused on children (Memmert, 2006; Neisser, 1979; Remington, Cartwright-Finch, \& Lavie, 2014). To our knowledge, only three published studies have examined IB in older adults, and only two of these compared IB in younger and older adults (Graham \& Burke, 2011; O'Shea \& Fieo, 2015; Stothart, Boot, \& Simons, 2015). Given that older adults exhibit cognitive decline in general attention-based tasks (see, e.g., Greenwood \& Parasuraman, 2004; Kramer, Humphrey, Larish, \& Logan, 1994; Tsang, 2013; Verhaeghen \& Cerella, 2002), as well as other failures of awareness, such as change blindness (Rizzo et al., 2009) and attentional blink (GeorgiouKaristianis et al., 2007), there is strong reason to believe that performance on IB tasks would be similarly affected by cognitive aging. 


\section{Inattentional blindness in older adults}

As we noted above, only three published studies have explored IB in older adults. In the first study, Graham and Burke (2011) compared IB rates in younger adults (1722 years) and older adults (61-81 years) using Simons and Chabris's (1999) "invisible gorilla" video, in which a black gorilla appears unexpectedly during an informal basketball game. Observers are required to selectively attend to one of two teams, which each comprise three players and are differentiated by their shirt color: white or black. Graham and Burke found that older adults were more likely than younger adults to miss the gorilla, regardless of which team they were tracking, but both groups were more likely to experience IB when they were tracking the white rather than the black players. The latter finding is consistent with previous research demonstrating that observers are more likely to detect an unexpected stimulus that matches their attentional set - that is, if it shares features with task-relevant items (Most et al., 2005; Most et al., 2001). Some limitations arise from the choice of task in Graham and Burke's (2011) study. For example, when using a staged video it is impossible to systematically manipulate the characteristics of the display in order to investigate the factors that influence IB, which could permit confounds between experimental conditions (Most et al., 2001). In addition, observers have no opportunity to practice the primary task, which can affect IB rates (Neisser, 1979; Richards et al., 2010). Because older adults are likely to be less familiar than college students with the types of primary tasks used in IB research (i.e., object tracking), this could artificially inflate older adults' IB rates. For these reasons, it would be optimal to compare younger and older adults' IB rates on a computerized task that permitted systematic manipulation of the key variables of interest, and provided participants with sufficient opportunity to practice the primary task before the unexpected stimulus was introduced.

More recently, O'Shea and Fieo (2015) used a computerized object-tracking IB task in a sample of 36 older adults $61-$ 79 years of age. The study did not include a young adult comparison group. The IB task involved five trials, which each lasted 11-17 s and comprised four white and four black letters moving on a gray background. Observers were required to track the black shapes on the first, third, and fourth trials, and were required to track the white shapes on the second trial. An additional black cross appeared on the third, fourth, and fifth trials. The third trial constituted the critical trial, in which the unexpected stimulus appeared for the first time. The fourth trial constituted the divided-attention trial, in which it was anticipated that participants would divide their attention between the primary task and searching for an unexpected stimulus, since the critical trial had alerted them that something unexpected might happen. For the fifth and final trial, observers were asked to watch the display without tracking objects; this provided a control condition, the full-attention trial, to ensure that the observers could detect the unexpected stimulus when they were not engaged in the primary task. $O$ 'Shea and Fieo found that nearly three-quarters of the older adults (74\%) experienced IB during the critical trial, despite the unexpected stimulus matching their attentional set for black objects. General fluid intelligence predicted IB rates, but processing speed did not (O'Shea \& Fieo, 2015).

Most recently, Stothart et al. (2015) explored IB within a large sample $(N=515)$ of adults ranging in age from 18 to 75 years. The researchers used a computerized objecttracking IB task and systematically manipulated the distance between observers' focus of attention and the location of the unexpected stimulus. Consistent with previous research (e.g., Graham \& Burke, 2011; Most, Simons, Scholl, \& Chabris, 2000) both age and distance from the focus of attention were associated with increased IB; however, they found no interaction between these factors (Stothart et al., 2015). A notable strength of this study was that it examined age as a continuous variable, with the results suggesting progressive decline in the probability of noticing throughout adulthood. Conversely, one limitation was that even though the overall sample size was very large, the proportion of adults 60 years of age and above was relatively small $(n=22)$.

\section{Age-related changes in cognition}

Whereas little is known about IB in older adults, a large literature has concentrated on age-related changes in attention and cognition more broadly. Several theories have been proposed to account for age-related changes in cognitive processing (Park \& McDonough, 2013). A comprehensive review is beyond the scope of the present article; however, some prominent theories pertain to processing speed (Salthouse, 1996), limited attentional capacity (Craik \& Byrd, 1982; Kahneman, 1973), and inhibitory deficits (Connelly, Hasher, \& Zacks, 1991; Lustig, Hasher, $\&$ Zacks, 2007). Although these theories were developed to explain performance in non-IB tasks, some of the theories might be applicable to predict age-related changes in IB rates.

Processing-speed theory proposes that slower cognitive processing is the major factor contributing to cognitive aging (Salthouse, 1996). Intended to explain age-related differences in fluid cognition, the evidence supporting processing-speed theory has come primarily from digit- and symbol-based tasks (e.g., arithmetic, pattern analysis, or copying). In simple tasks, performance differences manifest directly as differences in response speed. In more complex or hierarchical tasks, performance differences may also manifest as errors or completion failures, because cognitive slowing will impair the individual's ability to complete sequential and simultaneous operations. Although processing-speed theory is one of the dominant models of cognitive aging (Verhaeghen \& Cerella, 2002), its relevance to IB paradigms is less clear. One possibility is 
that cognitive slowing would impair primary-task performance, particularly on more complex dynamic IB tasks, although it is unclear whether this impairment would be associated with increased IB. For instance, Simons and Jensen (2009) found that individual differences in object-tracking speed did not predict IB, and as we noted earlier, previous research has shown no association between processing speed and IB in older adults (O'Shea \& Fieo, 2015). As such, other models of cognitive aging are arguably more relevant to predicting performance within the constraints of an IB task.

The attentional capacity model (Kahneman, 1973) and the related limited-resource theory (Craik \& Byrd, 1982) assume that attention is finite, placing a fixed limit on the individual's capacity to perform. Because different mental operations require varying levels of effort, when the amount of attentional capacity is insufficient for the task demands, performance will drop or information will not be processed (Kahneman, 1973). These models do not make explicit predictions about the detection or processing of unexpected stimuli; however, the implication is that unexpected information can only reach conscious awareness when the individual has sufficient attentional capacity to process both the primary task and the unexpected stimulus (Graham \& Burke, 2011). Thus, if older adults have reduced attentional capacity, as compared with younger adults, then they should show higher IB rates, which is consistent with previous research indicating that older adults are more susceptible to IB (Graham \& Burke, 2011; Stothart et al., 2015).

Whereas the theories described above predict greater IB with age, the inhibitory deficit model (Connelly et al., 1991; Lustig et al., 2007) suggests the opposite. This model also builds upon the notion that limited cognitive resources diminish as one ages, but it argues that reduced attentional capacity prevents older adults from effectively inhibiting irrelevant information (Kim, Hasher, \& Zacks, 2007). As inhibitory processes are diminished, irrelevant information is more likely to be processed, and consequently to distract the observer from the current task (Hasher, Lustig, \& Zacks, 2007; Kim et al., 2007). Empirical support for the inhibitory deficit model as come primarily from language-based tasks, including reading and problem solving (Connelly et al., 1991; Kim et al., 2007; Kramer et al., 1994). For example, older adults tend to read more slowly (Kim et al., 2007) and to show greater priming (Campbell, Grady, Ng, \& Hasher, 2012), due to their inability to inhibit distracting information. When applied to IB, the inhibitory deficit model suggests that older adults should exhibit reduced IB, assuming they will fail to inhibit the taskirrelevant unexpected stimulus. This is inconsistent with previous research comparing IB in younger and older adults, which showed increased IB in older adults (Graham \& Burke, 2011; Stothart et al., 2015). Thus, although older adults demonstrate inhibitory deficits across a range of tasks, especially contextual and language-based tasks, it is unclear whether these deficits extend to the unexpected, taskirrelevant stimuli that appear during IB paradigms.

\section{The present study}

We designed an experiment to explore age-related differences in IB, by comparing older and younger adults, as well as the factors that influence IB in older adults, by systematically manipulating task demands. Participants completed two IB tasks: one dynamic, one static. Most IB studies have included one trial that contained only a single unexpected stimulus, assuming that such a stimulus can only be truly unexpected once (Mack \& Rock, 1998; Wolfe, 1999). However, recent work has demonstrated that it is possible to induce IB repeatedly by using different unexpected events within the same task (Simons, 2010; Ward \& Scholl, 2015) or across different tasks (Beanland \& Pammer, 2010a; Kreitz, Furley, et al., 2015). Of particular relevance to the present study, Kreitz, Furley, et al. also administered static and dynamic IB tasks to the same participants within a single testing session and found no correlation between individuals' performance across the two tasks. This confirms that when key aspects of the task differ, as in the present study, it is valid to test the same participants with multiple IB tasks.

For our dynamic task, we used a computerized objecttracking task and systematically manipulated two factors that have been found to influence IB rates: the characteristics of the unexpected stimulus (i.e., whether it matched the observer's attentional set) and the presence of distractors (i.e., none vs. some). As we noted above, unexpected stimuli are more likely to be detected if they match the observer's attentional set. Previous IB research has suggested that there are two influential aspects of attentional set: features that observers set their attention to select (i.e., target items) and features that observers set their attention to ignore (i.e., distractors; see Most et al., 2005). Consequently, if participants are tracking black objects while ignoring white objects, they will be most likely to detect a black unexpected object and least likely to detect a white unexpected object, with other colors having intermediate detection rates (Most et al., 2001). Regarding distractor presence, Koivisto and Revonsuo (2008) found that IB increased from $22 \%$ with no distractors to $52 \%$ with distractors present. This implies that the presence of tobe-ignored distractors increases the task difficulty, which in turn increases IB (e.g., Cartwright-Finch \& Lavie, 2007). Interestingly, increasing the number of distractors (i.e., from one to five) did not impact IB rates, suggesting that the crucial factor is simply having something to ignore (Koivisto \& Revonsuo, 2008). We hypothesized that both findings would replicate in older adults, and that the relative effect sizes could provide insight into the mechanisms of IB. If IB rates are higher in older than in younger adults, especially when 
distractors are present, this would support attentional capacity models. In contrast, if IB rates for older adults are more affected by the match between the attentional set and the unexpected stimulus characteristics, this implies that inhibitory mechanisms mediate IB.

In addition to the dynamic task, we included a static cross judgment task, in which observers were briefly presented with a large cross and required to judge whether the horizontal or vertical arm of the cross was longer (Mack \& Rock, 1998). During the critical trial, a small shape appeared unexpectedly within one of the quadrants of the cross. Static tasks have been widely used in IB research (Cartwright-Finch \& Lavie, 2007; Mack \& Rock, 1998), including in developmental research on children (Remington et al., 2014), but never with older adults, so we included this task to explore whether similar patterns occurred across static and dynamic tasks. Given that previous research in IB (Graham \& Burke, 2011; Stothart et al., 2015), change blindness (Rizzo et al., 2009), and the attentional blink (Georgiou-Karistianis et al., 2007) had all shown that older adults were more susceptible than younger adults to failures of awareness, we set the parameters of the static task such that the younger adults were likely to have low IB rates, in order to provide greater scope for revealing increased IB in older adults.

\section{Method}

\section{Participants}

Overall, 166 adults participated, including 83 younger adults 18-25 years old and 83 older adults 60-80 years old. All of the participants had normal or corrected-to-normal visual acuity, as measured by a near-vision chart (viewing distance $40 \mathrm{~cm}$ ) and provided written informed consent. Younger adults who were enrolled in introductory psychology courses received course credit. Older adults went into a draw to win premium lounge cinema tickets. Ethical aspects of the research were approved by the Australian National University Human Research Ethics Committee.

The data for eight participants were excluded: three older adults achieved Montreal Cognitive Assessment (MoCA; Nasreddine et al., 2005) scores below 26; one older adult did not meet the age criteria; one older adult failed to complete all tasks; two younger adults failed to detect the unexpected stimulus under full attention; and one younger adult realized that it was an IB study. Participants were not excluded if they had some knowledge of IB but did not realize that they were participating in an IB study (Beanland \& Pammer, 2010a).

The final sample included in our analyses comprised 80 younger adults (52 female, 28 male) with a mean age of 20.7 years $(S D=2.4)$, and 78 older adults ( 58 female, 20 male) with a mean age of 69.6 years $(S D=5.1)$.

\section{Apparatus}

Stimuli were presented on a Dell Latitude E6420 15-in. laptop, with stimulus presentation and data acquisition controlled using PsychoPy (Peirce, 2007). The viewing distance was approximately $40 \mathrm{~cm}$.

\section{Dynamic object-tracking IB task}

The object-tracking task consisted of seven 20-s trials, preceded by a practice trial. The task demands were manipulated in a 2 (Number of Distractors: 0, 3) $\times 2$ (Unexpected Stimulus Color: black, white) between-subjects design. Participants were therefore randomly assigned to one of four dynamic task conditions: no distractors, black unexpected stimulus (19 younger adults, 19 older adults); no distractors, white unexpected stimulus (21 younger adults, 20 older adults); three distractors, black unexpected stimulus (20 younger adults, 19 older adults); or three distractors, white unexpected stimulus (20 younger adults, 20 older adults).

As is shown in Fig. 1, the display area consisted of a gray background $\left(24.8^{\circ} \times 18.7^{\circ}\right)$ with three target items that moved independently around the screen, occasionally bouncing off the display edges. Participants were required to track the attended target items, which were black circles subtending $1.4^{\circ} \times 1.4^{\circ}$, and to silently count the number of times they bounced during each trial (range: 15-19 bounces). The distractors, if present, were three white circles subtending $1.4^{\circ} \times 1.4^{\circ}$, which also moved

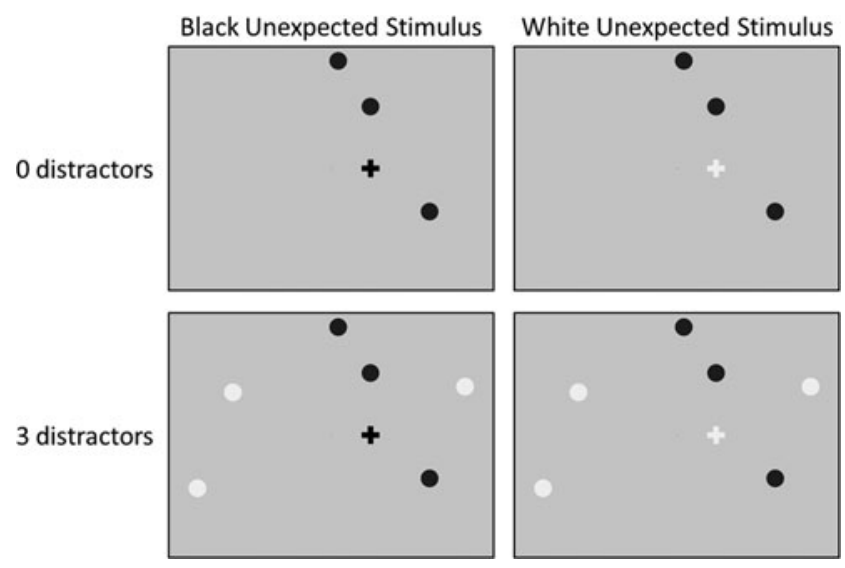

Fig. 1 Still frames from the dynamic object-tracking task, representing critical trials from each experimental condition. All trials contained three black circles, which were the attended target items that participants were required to track for $20 \mathrm{~s}$. The number of distractors (zero vs. three) was manipulated between subjects, so that half of the participants experienced no distractors and half experienced distractors, which were three white circles. During the critical trial, an additional, unexpected stimulus appeared: A cross, which was either black or white, appeared after $6 \mathrm{~s}$ and crossed the screen from right to left, exiting after a further $6 \mathrm{~s}$. The precritical trials $(1-4)$ employed the same configuration as the critical trials, except that no unexpected stimulus appeared 
independently and bounced at a similar rate to the attended items. The experimental sequence included four trial types: the precritical trials (Trials 1-4), critical trial (Trial 5), divided-attention trial (Trial 6), and full-attention trial (Trial 7).

The precritical trials contained only the expected items (i.e., only targets in the no-distractor conditions, or both targets and distractors in the three-distractor conditions) and provided observers an opportunity to practice the primary task. Participants were prompted to record the number of target item bounces immediately after each trial. We included four precritical trials after the practice phase because previous studies in our lab had shown that accuracy improved significantly during the first four trials and then plateaued (Beanland \& Pammer, 2012).

The critical, divided-attention, and full-attention trials contained an additional, unexpected stimulus: a cross that entered from the right display edge after $6 \mathrm{~s}$, and traversed the horizontal midline for $6 \mathrm{~s}$ before exiting to the left. The unexpected-stimulus color either matched (black) or did not match (white) the observer's attentional set. After reporting the bounce count for the critical trial, participants were asked whether they had noticed anything other than the expected items and, if so, to describe what they saw. Observers were classified as "noticers" if they detected the unexpected stimulus during the critical trial and could correctly report its color and/or shape. Participants then completed the dividedattention trial, which followed the same procedure as the critical trial. Finally, for the full-attention trial, participants were instructed to watch the display without tracking any of the objects, to ensure they could detect the unexpected stimulus when they were not engaging in the primary task. Participants who failed to detect the unexpected stimulus during the full-attention trial (two younger adults) were excluded from the data analyses.

\section{Static cross judgment IB task}

The cross judgment task was based on Mack and Rock's (1998) classic IB experiments, which has previously been adapted to explore age-related changes in IB among children and young adults (Remington et al., 2014). Our task consisted of eight experimental trials preceded by three practice trials. All of the stimuli were black on a light gray background. Each trial began with a small fixation point for $1,500 \mathrm{~ms}$, followed by $500 \mathrm{~ms}$ presentation of the stimulus cross, and then a pattern mask for 1,000 ms (see Fig. 2). Following the mask, participants were asked to report, via button press, whether the horizontal or vertical arm of the cross was longer. Trials 1-5 were precritical trials, which contained only the stimulus cross. Trials $6-8$ constituted

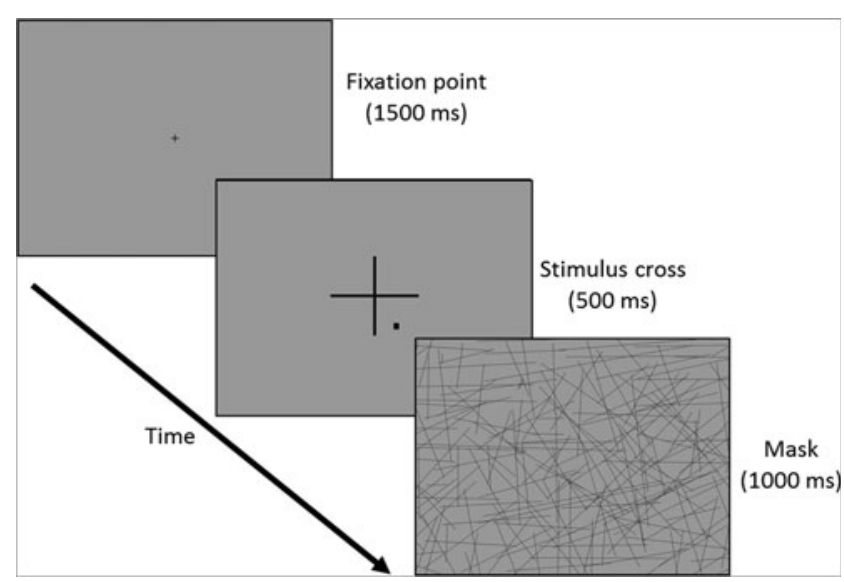

Fig. 2 Sequence of trials in the static cross judgment inattentional blindness task. The task comprised a total of eight trials. Trials 1-5 were precritical trials, which contained only the stimulus cross. The primary task was to determine whether the vertical or the horizontal arm of the cross was longer. An additional, unexpected stimulus (a small black square) appeared in the right-hand quadrant of the cross on Trials 6-8, which were, respectively, the critical trial, divided-attention trial, and full-attention trial. Observers who failed to detect the unexpected stimulus on the critical trial were deemed to have experienced inattentional blindness. The actual stimulus dimensions in the experiment were $0.25^{\circ} \times 0.25^{\circ}$ for the unexpected stimulus, and $3.22^{\circ}$ $\times 2.90^{\circ}$ for the cross during the critical trial

the critical trial, divided-attention trial, and full-attention trial, respectively. These included a small black square $\left(0.25^{\circ} \times 0.25^{\circ}\right)$ in the bottom right quadrant of the cross. Following the critical and divided-attention trials, participants were asked whether they had noticed anything in addition to the large cross on the preceding trial, and were classified as "noticers" if they could correctly report the shape or location of the unexpected stimulus. During the full-attention trial, participants were instructed to simply look at the display without judging the lengths of the cross arms. When they were no longer required to engage in the cross judgment task, all participants identified the unexpected stimulus.

\section{Procedure}

The participants were tested individually, seated comfortably in a quiet room. As a cover story, they were informed that the experiment was examining the impact of age on visual cognition. Prior to attempting the IB tasks, participants completed 20 trials of an object recognition task, in which they were required to identify whether a briefly presented image depicted an object or an animal. This task used the same general trial structure as the cross judgment task and was included to familiarize participants with the general format of computer-based experimental tasks. The picture identification task was deliberately easy, to reduce any stress older participants might experience. Participants then completed the 
dynamic IB task, followed by the static IB task. ${ }^{1}$ Finally, the older adults also completed the MoCA (Nasreddine et al., 2005), a brief clinical assessment of cognitive ability, language, and memory, in order to exclude participants with cognitive impairment. After completing the experimental tasks, the participants were debriefed and informed of the true experimental aims. During this debriefing, participants were asked whether they had previously been familiar with IB, and whether they had realized they were participating in an IB experiment. One participant who realized the experimental aims was excluded from the data analyses. Participants who did not realize the experimental aims were retained even if they were familiar with IB, consistent with previous research (Beanland \& Pammer, 2010a; Simons, 2010).

\section{Results}

\section{Dynamic object-tracking IB task}

Overall, $21 \%$ of participants experienced IB in the dynamic task, but IB rates varied substantially across the age groups and experimental conditions (see Fig. 3). Specifically, $38 \%$ of the older adults experienced IB, as compared with only $8 \%$ of the younger adults, $\chi^{2}(1)=21.52, p<.001$, odds ratio $(\mathrm{OR})=$ 7.7, $95 \%$ CI OR $[3.0,19.9]$.

Binary logistic regression was used to examine the associations between IB and the three independent variables. As was recommended by Hosmer, Lemeshow, and Sturdivant (2013), our initial model contained only main effects, assessing three categorical predictors (age, number of distractors, and unexpected stimulus color) for the dependent variable IB (i.e., failure to notice the unexpected stimulus). The reference groups were as follow: younger adults for age, zero for the number of distractors, and black for the unexpected stimulus color. Thus, ORs greater than 1 indicate that the comparison group (older adults, three distractors, or white unexpected stimulus) is associated with an increased likelihood of IB. Omnibus tests indicated that the full model was reliably different from the constant-only model, $\chi^{2}(3)=65.76, p<.001$, Nagelkerke $R^{2}$ $=.52$. All three variables independently predicted IB, with older age, distractor presence, and attentional set mismatch all increasing the probability of missing an unexpected

\footnotetext{
${ }^{1}$ Given that previous research had demonstrated that it is possible to induce IB repeatedly using different IB tasks and/or different unexpected events (e.g., Beanland \& Pammer, 2010a; Kreitz, Furley, et al., 2015; Simons, 2010; Ward \& Scholl, 2015), a study including two distinct IB tasks should obtain equivalent results, regardless of whether the task presentation order is fixed or counterbalanced. However, since we were most interested in performance in the dynamic task, we chose to use a fixed order with the dynamic task first, and to conduct statistical analyses to explore whether observers' expectations affected IB rates during the static task.
}

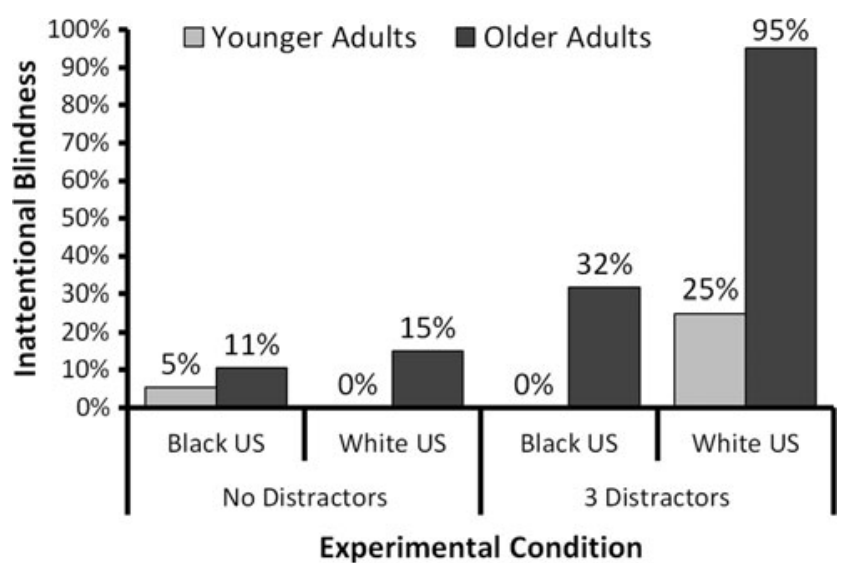

Fig. 3 Rates of inattentional blindness in the dynamic object-tracking task, by experimental condition and age group. The attended items were black circles, and the distractors were white circles

stimulus (see Table 1). The model correctly classified $89 \%$ of participants ( $99 \%$ of noticers, $53 \%$ of nonnoticers).

Interactions were added to the main-effects model one at a time, as per Hosmer et al.'s (2013) guidelines. The three-way interaction $($ Age $\times$ Distractor Presence $\times$ Unexpected Stimulus Color) could not be reliably assessed, since it contained multiple zero cells (i.e., two experimental conditions in which no young adults experienced IB). Nonsignificant improvements in model fit indicated that age did not significantly interact with either distractor presence $\left(\chi^{2}=0.79, p=.375\right)$ or unexpected stimulus color $\left(\chi^{2}=0.09, p=.759\right)$. Conversely, the model fit was significantly improved by adding the interaction between number of distractors and unexpected stimulus color, $\chi^{2}=8.00, p=.005$. The final model including the interaction term is shown in Table 1. After adding the interaction term, age remained significantly associated with IB rates; however, the main effects for distractor presence and unexpected stimulus color were no longer statistically significant, and the OR for unexpected stimulus color reversed direction (i.e., from $>1$ in the model that included only main effects, to $<1$ in the model including the interaction term). This suggests that attentional set mismatch is less influential in IB tasks that do not incorporate to-be-ignored distractors. Consistent with this, pairwise comparisons between the specific experimental conditions revealed that attentional set mismatch was associated with IB in the three-distractor conditions (IB: $15 \%$ vs. $60 \%$, $\chi^{2}=16.69, p<.001$, OR 8.3, $95 \%$ CI OR $\left.[2.8,24.2]\right)$, but not in the two no-distractor conditions (IB: $8 \%$ vs. $7 \% ; \chi^{2}=0.01$, $p=1.00$, OR $0.9,95 \%$ CI OR $[0.2,4.9])$. Distractor presence was associated with a nonsignificant increase in IB when the unexpected stimulus matched the observers' attentional set (IB: $8 \%$ vs. $15 \%, \chi^{2}=1.05, p=.481$, OR $2.1,95 \% \mathrm{CI}$ OR $[0.5,9.2])$, but a significant increase when the unexpected stimulus did not match the observer's attentional set (IB: $7 \%$ vs. $60 \%, \chi^{2}=25.3, p<.001$, OR 19.0, $95 \%$ CI OR [5.0, 72.2]). 
Table 1 Logistic regression results: Predictors of inattentional blindness in the dynamic object-tracking task

\begin{tabular}{|c|c|c|c|c|c|c|}
\hline \multirow[t]{2}{*}{ Parameter } & \multirow[t]{2}{*}{$B$} & \multirow[t]{2}{*}{$S E$} & \multirow[t]{2}{*}{ Wald $\chi^{2}$} & \multirow[t]{2}{*}{ Odds Ratio } & \multicolumn{2}{|c|}{$95 \%$ CI Odds Ratio } \\
\hline & & & & & Lower & Upper \\
\hline \multicolumn{7}{|l|}{ Model 1: Main Effects Only } \\
\hline Age group & 2.84 & 0.62 & $21.08^{* * *}$ & 17.2 & 5.1 & 57.8 \\
\hline Number of distractors & 2.83 & 0.62 & $20.82^{* * *}$ & 17.0 & 5.0 & 57.5 \\
\hline US color & 2.12 & 0.59 & $13.17^{* * *}$ & 8.4 & 2.7 & 26.3 \\
\hline (Constant) & -6.10 & 1.00 & $37.29^{* * *}$ & & & \\
\hline \multicolumn{7}{|c|}{ Model 2: Full Model with Main Effects and Interaction } \\
\hline Age group & 3.47 & 0.82 & $17.90^{* * *}$ & 32.0 & 6.4 & 159.3 \\
\hline Number of distractors & 0.88 & 0.79 & 1.23 & 2.4 & 0.5 & 11.3 \\
\hline US color & -0.06 & 0.88 & 0.01 & 0.9 & 0.2 & 5.3 \\
\hline Distractors $\times$ US color & 3.40 & 1.23 & $7.68^{* *}$ & 30.0 & 2.7 & 332.3 \\
\hline (Constant) & -5.18 & 1.00 & $26.66^{* * *}$ & & & \\
\hline
\end{tabular}

US = unexpected stimulus. The reference groups were: young adults for age group; 0 for number of distractors; and black for US color. ${ }^{* *} p<.01,{ }^{* * *} p<.001$

Proportional object-tracking error rates were calculated using the formula (actual - reported bounces)/actual bounces. Because some participants overreported the number of bounces, the absolute proportional error score was used for the analyses. As is shown in Fig. 4, error rates were highest for the first three precritical trials, with older adults performing nonsignificantly worse than younger adults [Precritical Trial 1: $M_{\text {diff }}=.05, t(156)=1.30, p=.197, d=0.21$; Precritical Trial 2: $M_{\text {diff }}=.04, t(156)=1.19, p=.235, d=0.19$; Precritical Trial 3: $M_{\text {diff }}=.03, t(156)=1.12, p=.266, d=$ $0.18]$. These patterns suggest that some participants, especially older adults, required a longer practice phase. Performance on the fourth precritical trial (i.e., the final trial before the unexpected stimulus appeared during the critical trial) was equivalent for younger and older adults, $M_{\mathrm{diff}}=.003, t(156)$ $=0.11, p=.909, d=0.02$.

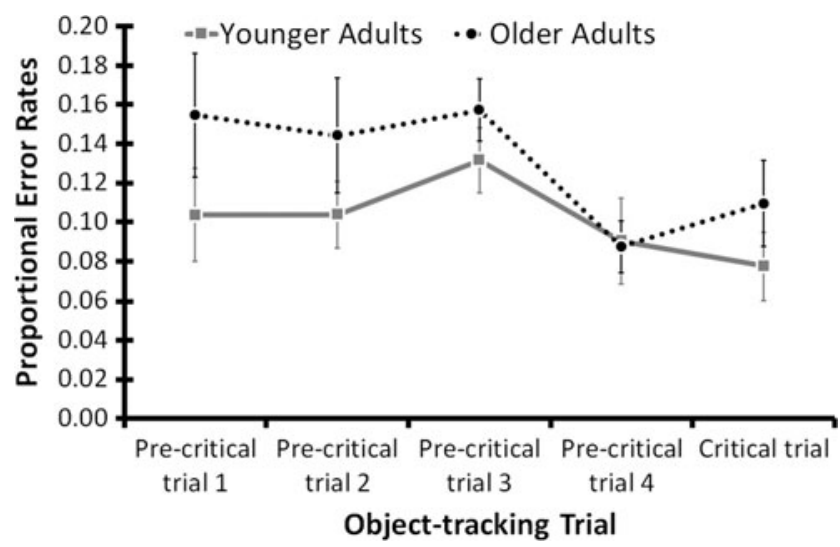

Fig. 4 Proportional error rates for each trial of the dynamic objecttracking task, by age group. Higher scores indicate greater errors. Error bars represent \pm 1 standard errors of the means
Error rates for the critical trial were analyzed using a 2 (IB: yes, no) $\times 2$ (Age Group: younger adults, older adults $) \times 2$ (Distractors: 0,3$)$ mixed analysis of variance. Consistent with several previous studies (e.g., Beanland \& Pammer, 2010b; Simons \& Jensen, 2009), no main effect of IB emerged, $F(1$, $150)=0.00, p=.999, \eta_{\mathrm{p}}{ }^{2}=.00$. The mean error rates were almost identical for noticers $(M=.08, S E=.02)$ and nonnoticers $(M=.08, S E=.05)$. As with the precritical trials, we found no significant difference in error rates between younger $(M=.06, S E=.05)$ and older $(M=.11, S E=.03)$ adults, $F(1,150)=0.77, p=.380, \eta_{\mathrm{p}}{ }^{2}=.01$. We also found no significant effect of distractor presence on error rates, $F(1,150)=$ $0.34, p=.559, \eta_{\mathrm{p}}{ }^{2}=.00$ (no distractors: $M=.07, S E=.05$; three distractors: $M=.10, S E=.03)$. Finally, none of the interactions were statistically significant (all $F \mathrm{~s}<0.5, p \mathrm{~s}>.49$ ).

\section{Static cross judgment IB task}

Overall, $46 \%$ of the participants experienced IB, but the proportion varied significantly with age: The vast majority of older adults (89\%) experienced IB, but very few younger adults $(5 \%)$ did, $\chi^{2}(1)=110.68, p<.001$, OR $=145.7$, $95 \%$ CI OR [42.9, 494.4].

Nonparametric tests revealed that the average cross judgment accuracy across the four precritical trials was significantly higher for younger $(M=85 \%, S D=28 \%, \mathrm{Mdn}=100 \%)$ than for older $(M=81 \%, S D=21 \%$, Mdn $=80 \%)$ adults, MannWhitney $U=2,525, z=-2.28, p=.023$. The average accuracies across the four precritical trials did not differ between noticers $(M=84 \%, S D=28 \%, M d n=100 \%)$ and nonnoticers $(M=$ $82 \%, S D=20 \%, \operatorname{Mdn}=100 \%$ ) of the unexpected stimulus, $U$ $=2,702, z=-1.54, p=.124$. Accuracies on the critical trial did not differ between older and younger adults $(U=2,911, z=-$ 
$1.19, p=.265)$ or between noticers and nonnoticers of the unexpected stimulus $(U=2,915, z=-1.07, p=.366)$.

It is worth noting that the difference in IB rates between older and younger adults was largest for the static task. Because the participants completed the dynamic task prior to the static task, one possibility is that participants may have possessed an implicit expectation that another unexpected stimulus would appear during the static task. Given that the younger adults were probably more familiar than older adults with the format of experimental tasks (many of the younger adults were enrolled in introductory psychology courses), this expectation could have affected the two age groups differentially. To assess whether this could have biased our results, we reran the static-task analyses including only those participants who scored $100 \%$ on all trials of the cross judgment task, on the assumption that participants with an implicit expectation that something else might happen would be less attentive, and therefore potentially less accurate, on the primary task. This subsample of perfectly accurate observers $(n=79)$ comprised 46 younger adults and 33 older adults. IB remained significantly higher for the older (88\%) than for the younger $(9 \%)$ adults, $\chi^{2}(1, N=79)=49.53, p<.001$, OR 76.1, $95 \%$ CI OR $[17.6,329.3]$, suggesting that differential expectations in the static task cannot explain older adults' disproportionately higher IB rate. Rather, the fact that primary-task accuracies differed in the static but not in the dynamic task suggests that the relative difficulty for older versus younger adults was greater in the cross judgment task than in the object-tracking task.

\section{Within-subjects IB rates}

Administering two IB tasks to the same sample permitted us to explore within-subjects rates of IB, which is a topic that has received very little research to date (Kreitz, Furley, et al., 2015). There was a moderately sized significant correlation between IB on the two tasks, $r(158)=.37, p<.001,95 \%$ CI $[.23, .50]$. Closer inspection of the data revealed that this correlation was due to age differences, since most younger adults $(87.5 \%)$ detected the unexpected stimulus in both tasks, whereas a large proportion of the older adults $(37 \%)$ experienced IB in both tasks. Within the younger adult group, no correlation was apparent between IB on the two tasks, $r(80)=-.07, p=.565,95 \% \mathrm{CI}[-.11,-.03]$. All young adult observers who experienced IB during the static task had been noticers during the dynamic task, whereas all young adults who experienced IB during the dynamic task had been noticers in the static task. Among older adults, we observed a small, nonsignificant correlation between IB on the two tasks, $r(78)=.20, p=.075,95 \%$ CI $[.01, .33]$; the rate of IB in the static task was $97 \%$ for those who experienced IB during the dynamic task, as compared with $83 \%$ for observers who had noticed the dynamic unexpected stimulus.

\section{Discussion}

The primary result of the present study is that IB is vastly more common in older adults (60-80 years) than in young adults (18-25 years) across a range of experimental tasks. These findings are consistent with previous research indicating that IB rates increase in older adulthood (Graham \& Burke, 2011; Stothart et al., 2015). Thus, when considered alongside previous research, the present results demonstrate that older adults experience a widespread decline in their ability to detect unexpected objects across a range of task demands. Indeed, it is possible that our results understate the magnitude of the difference between older and young adults, given that noticing rates were at ceiling $(\leq 5 \% \mathrm{IB})$ for younger adults in all but one of the conditions we tested.

In the dynamic IB task, age had a strong main effect on IB rates but did not interact with experimental condition. The lack of a significant interaction effect is consistent with Stothart et al.'s (2015) research, which also showed that age did not interact with experimental condition. We observed a significant interaction between distractor presence and attentional set mismatch of the unexpected stimulus: IB rates were lowest for both age groups in the absence of to-be-ignored distractors, and were highest when distractors were present and physically similar to the unexpected stimulus. Notably, the unexpected-stimulus color only affected IB rates when distractors were present. This suggests that observers adopt a less focused attentional set when distractors are absent, whereas the presence of to-be-ignored distractors requires a more focused attentional set, in order to select only attended items and suppress distractors. The fact that only $5 \%$ of older adults detected a white unexpected stimulus when they had an attentional set to attend to black targets and ignore white distractors suggests that they were fully capable of inhibiting irrelevant information within an IB task, which in turn demonstrates that the inhibitory deficit model cannot account for age-related changes in IB.

Another aspect of the results that contradicts the inhibitory deficit model is the fact that adding distractors did not influence older adults' primary-task performance; if inhibitory deficits did influence performance in dynamic IB tasks, the presence of distractors should have impaired older adults' object-tracking performance. Interestingly, although IB rates varied markedly with age, older adults were not significantly worse in terms of primary-task accuracy. This seems most consistent with attentional capacity results of cognitive aging: Older adults had sufficient capacity to complete the primary task to the same level as younger adults, but were less able to detect an unexpected stimulus. One explanation is that older adults' reduced cognitive resources forced them to adopt a more focused attentional set, such that they selectively attended only to the target items, which resulted in failure to consciously process stimuli that fell outside of this attentional 
set. This included both distractor items (which, as noted, did not affect primary-task performance) and unexpected objects.

In the static IB task, age was again a significant predictor of IB, with $89 \%$ of older adults missing the unexpected stimulus, as compared with only $5 \%$ of younger adults. Accuracy differed as a function of age group, suggesting that the task was relatively more demanding for older participants. Again, this finding is most consistent with attentional capacity models of cognitive aging, since older adults found the primary task more challenging and were much less likely to detect the unexpected object, suggesting that they had insufficient cognitive resources to process the unexpected black square when they were concurrently undertaking the cross judgment task. Interestingly, previous research using a similar task in children and young adults (Remington et al., 2014) had revealed similarly low rates of IB in young adults, with significantly higher rates for children. Taken together, this suggests that there may be a U-shaped relationship between IB and age, with IB rates declining from childhood to young adulthood, and then increasing again in older adulthood.

The increase in IB susceptibility with age could have profound practical implications, given that IB is analogous to real-world "looked-but-failed-to-see" errors (Beanland, Lenné, \& Rößger, 2015), which account for around $5 \%$ of road crashes (Beanland, Fitzharris, Young, \& Lenné, 2013; Stutts, Reinfurt, Staplin, \& Rodgman, 2001). Empirical research has consistently demonstrated that older adults have disproportionately high crash rates and are at greater risk of injury, due to physical vulnerabilities and cognitive limitations (e.g., Anstey, Wood, Lord, \& Walker, 2005; Chaparro, Wood, $\&$ Carberry, 2004). Our results raise the prospect that older adults could have a particular impairment in detecting unexpected objects, which in real-world settings could increase their risks of both road crashes (e.g., due to failure to detect hazards) and falls (e.g., if they tripped over an unnoticed obstacle). As such, in future research it would be worthwhile exploring the extent to which age-related increases in IB generalize to real-world tasks, including driving and walking.

Although the present study adds to the existing literature on IB in older adults, by demonstrating that robust agerelated increases in IB occur across a range of tasks, a couple of methodological limitations should be noted. First, we obtained very low rates of IB in our younger adult sample, because we simplified our tasks to make them easier for older adults (e.g., slower speeds and a reduced number of items in the dynamic task, and a longer presentation time and larger unexpected stimulus in the static task). This may have obscured the true extent of age-related differences in IB, which could be better identified with a task that yielded higher IB rates in younger adults. However, this would risk the reverse problem occurring (i.e., $100 \%$ IB in the older adults), so in practice this limitation may be challenging to avoid in future research.
A second limitation was that, though our total sample was relatively large, in the dynamic task we had only 19-21 participants per condition. Although this is similar to the cell sizes employed in many previous IB studies (e.g., Most et al., 2005; Most et al., 2001), it limits statistical power. Thus age-related interactions might not have been reliably detected in our sample. Another issue arising from the sample size is reliability: Our age-related effects were large, but also the $95 \%$ confidence intervals for these effect sizes were wide. This was due to the fact that IB paradigms produce dichotomous outcomes, classifying individuals as "noticers" or "nonnoticers" on the basis of a single measurement (i.e., one critical trial), which is a fairly unreliable measure (Beanland \& Chan, in press). Although it is possible to induce IB repeatedly across different stimuli and tasks, as has been shown in both the present study and previous research (e.g., Beanland \& Pammer, 2010a; Kreitz, Furley, et al., 2015; Simons, 2010; Ward \& Scholl, 2015), it is much more difficult to induce IB repeatedly using identical stimuli. In the absence of a proper repeated measures design, the best way to yield reliable experimental results is to test larger sample sizes.

One possibility for increasing sample sizes would be to run studies online, which has yielded large samples for some IB studies (e.g., Brown-Iannuzzi, Hoffman, Payne, \& Trawalter, 2014; Kreitz, Schnuerch, Gibbons, \& Memmert, 2015; Stothart et al., 2015; Ward \& Scholl, 2015). However, as Stothart et al. noted, online studies may have even greater difficulties than face-to-face studies in recruiting older participants. Another issue is that cognitive screening assessments, such as the MoCA, cannot be reliably administered online. As such, the most pragmatic approach to studying IB in older adults may be to incorporate IB tasks into broader studies of cognitive aging, for which large samples have already been recruited and screened for eligibility.

In conclusion, the present study demonstrates that older adults are significantly more likely to experience IB than younger adults across a range of experimental conditions. The results presented are best accounted for by the attentional capacity model of cognitive aging, which suggests that adults' attentional resources diminish with age, preventing unexpected or irrelevant items from being fully processed. In future studies, it would be instructive to recruit samples in which age could be set as a continuous, rather than a categorical, variable, to explore whether IB susceptibility increases continually as a function of age, or rather as a function of particular factors that arise when an adult reaches a critical age.

Author note This research was conducted as part of an Australian Research Council Linkage Project (No. LP130100181) and comprised part of S.H.'s honors thesis at the Australian National University. V.B. is supported by an Australian Research Council Discovery Early Career Researcher Award (No. DE150100083). We thank Ann Plummer for assistance with data collection. 


\section{References}

Anstey, K. J., Wood, J., Lord, S., \& Walker, J. G. (2005). Cognitive, sensory and physical factors enabling driving safety in older adults. Clinical Psychology Review, 25, 45-65.

Beanland, V., \& Chan, E. H. C. (in press). The relationship between sustained inattentional blindness and working memory capacity. Attention, Perception, \& Psychophysics. doi:10.3758/s13414-0151027-x

Beanland, V., Fitzharris, M., Young, K. L., \& Lenné, M. G. (2013). Driver inattention and driver distraction in serious casualty crashes: Data from the Australian National Crash In-Depth Study. Accident Analysis and Prevention, 54, 99-107. doi:10.1016/j.aap.2012.12. 043

Beanland, V., Lenné, M. G., \& Rößger, L. (2015). Psychological factors in seeing motorcycles. In L. Rößger, M. G. Lenné, \& G. Underwood (Eds.), Increasing motorcycle conspicuity: Design and assessment of interventions to enhance rider safety (pp. 21-49). Aldershot, UK: Ashgate.

Beanland, V., \& Pammer, K. (2010a). Gorilla watching: Effects of exposure and expectations on inattentional blindness. In W. Christensen, E. Schier, \& J. Sutton (Eds.), ASCS09: Proceedings of the 9th Conference of the Australasian Society for Cognitive Science (pp. 12-20). Sydney, Australia: Macquarie Centre for Cognitive Science.

Beanland, V., \& Pammer, K. (2010b). Looking without seeing or seeing without looking? Eye movements in sustained inattentional blindness. Vision Research, 50, 977-988. doi:10.1016/j.visres.2010.02. 024

Beanland, V., \& Pammer, K. (2012). Minds on the blink: The relationship between inattentional blindness and attentional blink. Attention, Perception, \& Psychophysics, 74, 322-330. doi:10.3758/s13414011-0241-4

Bredemeier, K., \& Simons, D. (2012). Working memory and inattentional blindness. Psychonomic Bulletin \& Review, 19, 239-244. doi:10. 3758/s13423-011-0204-8

Brown-Iannuzzi, J. L., Hoffman, K. M., Payne, B. K., \& Trawalter, S. (2014). The invisible man: Interpersonal goals moderate inattentional blindness to African Americans. Journal of Experimental Psychology: General, 143, 33-37. doi:10.1037/ a0031407

Campbell, K. L., Grady, C. L., Ng, C., \& Hasher, L. (2012). Age differences in the frontoparietal cognitive control network: Implications for distractibility. Neuropsychologia, 50, 2212-2223. doi:10.1016/j. neuropsychologia.2012.05.025

Cartwright-Finch, U., \& Lavie, N. (2007). The role of perceptual load in inattentional blindness. Cognition, 102, 321-340. doi:10.1016/j. cognition.2006.01.002

Chaparro, A., Wood, J. M., \& Carberry, T. (2004). Effects of age and auditory and visual dual-tasks on closed road driving performance. Proceedings of the Human Factors and Ergonomics Society Annual Meeting, 48, 2319-2322. doi:10.1177/154193120404801924

Connelly, L. S., Hasher, L., \& Zacks, R. T. (1991). Age and reading: The impact of distraction. Psychology and Aging, 6, 533-541. doi:10. 1037/0882-7974.6.4.533

Craik, F. I. M., \& Byrd, M. (1982). Aging and cognitive deficits. In F. I. M. Craik \& S. Trehub (Eds.), Aging and cognitive processes (pp. 191-211). New York, NY: Springer.

Georgiou-Karistianis, N., Tang, J., Vardy, Y., Sheppard, D., Evans, N., Wilson, M., . . Bradshaw, J. (2007). Progressive age-related changes in the attentional blink paradigm. Aging, Neuropsychology, and Cognition, 14, 213-226. doi:10.1080/13825580500320681

Graham, E. R., \& Burke, D. M. (2011). Aging increases inattentional blindness to the gorilla in our midst. Psychology and Aging, 26, 162-166. doi:10.1037/a0020647
Greenwood, P. M., \& Parasuraman, R. (2004). The scaling of spatial attention in visual search and its modification in healthy aging. Perception \& Psychophysics, 66, 3-22. doi:10.3758/BF03194857

Grossman, E. S., Hoffman, Y. S. G., Berger, I., \& Zivotofsky, A. Z. (2015). Beating their chests: University students with ADHD demonstrate greater attentional abilities on an inattentional blindness paradigm. Neuropsychology, 29, 882-887. doi:10.1037/ neu0000189

Hannon, E. M., \& Richards, A. (2010). Is inattentional blindness related to individual differences in visual working memory capacity or executive control functioning? Perception, 39, 309-319. doi:10.1068/p6379

Hasher, L., Lustig, C., \& Zacks, R. (2007). Inhibitory Mechanisms and the Control of Attention. In A. R. A. Conway, C. Jarrold, M. J. Kane, A. Miyake, \& J. N. Towse (Eds.), Variation in working memory (1st ed., pp. 227-249). Oxford, UK: Oxford University Press.

Hosmer, D. W., Lemeshow, S., \& Sturdivant, R. X. (2013). Applied logistic regression (3rd ed.). New York, NY: Wiley.

Kahneman, D. (1973). Attention and effort. Hoboken, NJ: Prentice-Hall.

Kim, S., Hasher, L., \& Zacks, R. T. (2007). Aging and a benefit of distractibility. Psychonomic Bulletin \& Review, 14, 301-305. doi: 10.3758/BF03194068

Koivisto, M., \& Revonsuo, A. (2008). The role of unattended distractors in sustained inattentional blindness. Psychological Research, 72, 39-48. doi:10.1007/s00426-006-0072-4

Kramer, A. F., Humphrey, D. G., Larish, J. F., \& Logan, G. D. (1994). Aging and inhibition: Beyond a unitary view of inhibitory processing in attention. Psychology and Aging, 9, 491-512. doi:10.1037/ 0882-7974.9.4.491

Kreitz, C., Furley, P., Memmert, D., \& Simons, D. J. (2015a). Inattentional blindness and individual differences in cognitive abilities. PLoS ONE, 10, e0134675. doi:10.1371/journal.pone.0134675

Kreitz, C., Schnuerch, R., Gibbons, H., \& Memmert, D. (2015b). Some see it, some don't: Exploring the relation between inattentional blindness and personality factors. PLOS ONE, 10, e0128158. doi: 10.1371/journal.pone. 0128158

Lustig, C., Hasher, L., \& Zacks, R. (2007). Inhibitory deficit theory: Recent developments in a "new view.". In C. M. MacLeod \& D. S. Gorfein (Eds.), Inhibition in cognition (pp. 145-162). Washington, DC: American Psychological Association. doi:10. 1037/11587-008

Mack, A., \& Rock, I. (1998). Inattentional blindness. Cambridge, MA: MIT Press.

Memmert, D. (2006). The effects of eye movements, age, and expertise on inattentional blindness. Consciousness and Cognition, 15, 620 627. doi:10.1016/j.concog.2006.01.001

Most, S. B., Scholl, B. J., Clifford, E. R., \& Simons, D. J. (2005). What you see is what you set: Sustained inattentional blindness and the capture of awareness. Psychological Review, 112, 217-242. doi:10. 1037/0033-295X.112.1.217

Most, S. B., Simons, D. J., Scholl, B. J., \& Chabris, C. F. (2000). Sustained inattentional blindness: the role of location in the detection of unexpected dynamic events. Psyche: An Interdisciplinary Journal of Research on Consciousness, 6(14).

Most, S. B., Simons, D. J., Scholl, B. J., Jimenez, R., Clifford, E., \& Chabris, C. F. (2001). How not to be seen: The contribution of similarity and selective ignoring to sustained inattentional blindness. Psychological Science, 12, 9-17. doi:10.1111/1467-9280.00303

Nasreddine, Z. S., Phillips, N. A., Bédirian, V., Charbonneau, S., Whitehead, V., Collin, I., . . . Chertkow, H. (2005). The Montreal Cognitive Assessment, MoCA: A brief screening tool for mild cognitive impairment. Journal of the American Geriatrics Society, 53, 695-699. doi:10.1111/j.1532-5415.2005.53221.x

Neisser, U. (1979). The control of information pickup in selective looking. In A. D. Pick (Ed.), Perception and its development: A tribute to Eleanor J. Gibson (pp. 201-219). Hillsdale, NJ: Erlbaum. 
O'Shea, D. M., \& Fieo, R. A. (2015). Individual differences in fluid intelligence predicts inattentional blindness in a sample of older adults: A preliminary study. Psychological Research, 79, 570-575. doi:10.1007/s00426-014-0594-0

Park, D. C., \& McDonough, I. M. (2013). The dynamic aging mind: Revelations from functional neuroimaging research. Perspectives on Psychological Science, 8, 62-67. doi:10.1177/1745691612469034

Peirce, J. W. (2007). PsychoPy-Psychophysics software in Python. Journal of Neuroscience Methods, 162, 8-13. doi:10.1016/j. jneumeth.2006.11.017

Remington, A., Cartwright-Finch, U., \& Lavie, N. (2014). I can see clearly now: The effects of age and perceptual load on inattentional blindness. Frontiers in Human Neuroscience, 8, 229. doi:10.3389/ fnhum.2014.00229

Richards, A., Hannon, E. M., \& Derakshan, N. (2010). Predicting and manipulating the incidence of inattentional blindness. Psychological Research, 74, 513-523. doi:10.1007/s00426-009-0273-8

Rizzo, M., Sparks, J., McEvoy, S., Viamonte, S., Kellison, I., \& Vecera, S. P. (2009). Change blindness, aging, and cognition. Journal of Clinical and Experimental Neuropsychology, 31, 245-256. doi:10. 1080/13803390802279668

Salthouse, T. A. (1996). The processing-speed theory of adult age differences in cognition. Psychological Review, 103, 403-428. doi:10. 1037/0033-295x.103.3.403

Seegmiller, J. K., Watson, J. M., \& Strayer, D. L. (2011). Individual differences in susceptibility to inattentional blindness. Journal of Experimental Psychology: Learning, Memory, and Cognition, 37, 785-791. doi:10.1037/a0022474

Simons, D. J. (2010). Monkeying around with the gorillas in our midst: Familiarity with an inattentional-blindness task does not improve the detection of unexpected events. i-Perception, 1, 3-6. doi:10.1068/i0386
Simons, D. J., \& Chabris, C. F. (1999). Gorillas in our midst: Sustained inattentional blindness for dynamic events. Perception, 28, 1059 1074. doi:10.1068/p2952

Simons, D. J., \& Jensen, M. S. (2009). The effects of individual differences and task difficulty on inattentional blindness. Psychonomic Bulletin \& Review, 16, 398-403. doi:10.3758/ pbr.16.2.398

Stothart, C. R., Boot, W. R., \& Simons, D. J. (2015). Using Mechanical Turk to assess the effects of age and spatial proximity on inattentional blindness. Collabra, 1, 2. doi:10. 1525/collabra.26

Stutts, J. C., Reinfurt, D. W., Staplin, L., \& Rodgman, E. A. (2001). The role of driver distraction in traffic crashes. Washington, DC: AAA Foundation for Traffic Safety.

Swettenham, J., Remington, A., Murphy, P., Feuerstein, M., Grim, K., \& Lavie, N. (2014). Seeing the unseen: Autism involves reduced susceptibility to inattentional blindness. Neuropsychology, 28, 563570. doi:10.1037/neu0000042

Tsang, P. S. (2013). Ageing and attentional control. Quarterly Journal of Experimental Psychology, 66, 1517-1547. doi:10.1080/17470218. 2012.752019

Verhaeghen, P., \& Cerella, J. (2002). Aging, executive control, and attention: A review of meta-analyses. Neuroscience and Behavioural Reviews, 26, 849-857. doi:10.1016/S0149-7634(02)00071-4

Ward, E. J., \& Scholl, B. J. (2015). Inattentional blindness reflects limitations on perception, not memory: Evidence from repeated failures of awareness. Psychonomic Bulletin \& Review, 22, 722-727. doi:10. 3758/s13423-014-0745-8

Wolfe, J. M. (1999). Inattentional amnesia. In V. Coltheart (Ed.), Fleeting memories: Cognition of brief visual stimuli (pp. 71-94). Cambridge, MA: MIT Press. 